\title{
Stretchy robots snap shut
}

\author{
Adv. Funct. Mater. 1906603 (2019)
}

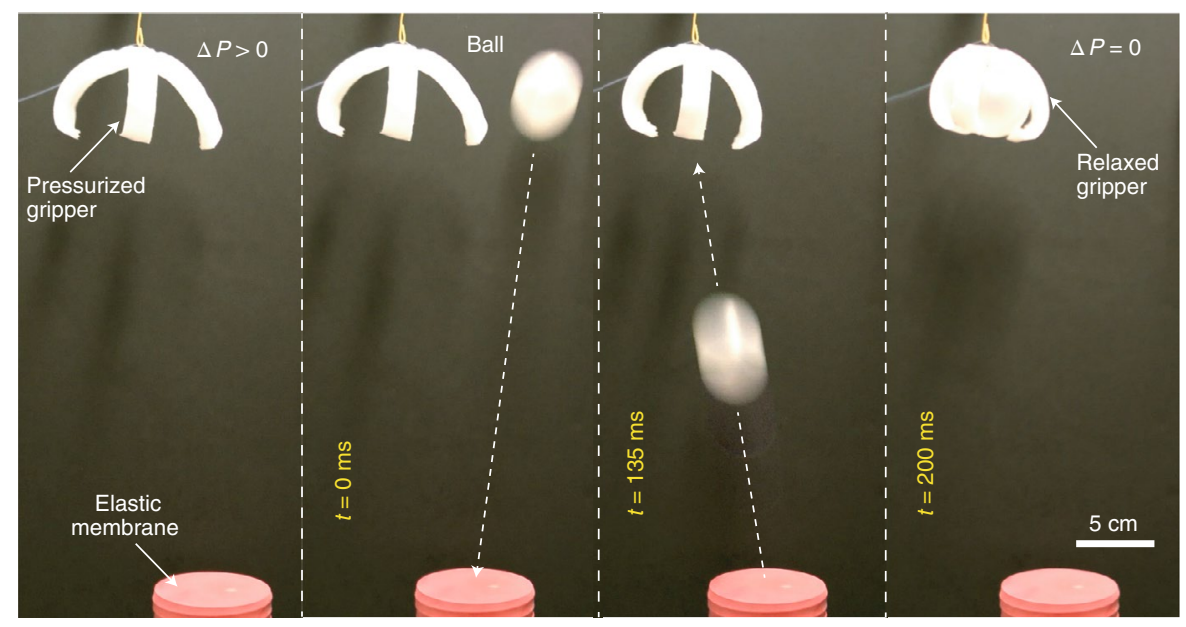

Credit: Wiley

Completely soft robots require energy sources that are themselves soft and can be efficiently applied to actuate the robot. Many plants and animals, from Venus flytraps to kangaroos, use elastic energy stored as strain, which is not only efficient but can also be released rapidly. However, realizing this in soft machines has been difficult due to the absence of soft but robust materials. Ramses Martinez and colleagues at Purdue University have now developed a programmable prestressed soft actuator that can store and release elastic energy.

The researchers fabricate the soft actuators by combining a flexible but non-stretchy material, such as paper, with an elastomer in a stressed state. In its relaxed state, the strain causes the actuator to curl, which can be reversed by inflating a pneumatic channel in the elastomer. Soft grippers in the relaxed state were able to hold payloads a hundred times their own weight without any external power. The stored elastic energy in the pressurized state can restore the soft actuator form within tens of milliseconds, allowing, for example, high-speed catching. Other systems were designed where the direction and strength of the prestressing allowed the researchers to program sequences of motion from a single channel.

Matthew Parker

Published online: 18 November 2019 https://doi.org/10.1038/s41928-019-0336-9 